University of Wollongong

Research Online

Faculty of Engineering and Information

Faculty of Engineering and Information

Sciences - Papers: Part A

Sciences

$1-1-2014$

Intelligent control of electroactive polymer actuators based on fuzzy and neurofuzzy methodologies

Christopher M. Druitt

University of Wollongong, cmd096@uowmail.edu.au

Gursel Alici

University of Wollongong, gursel@uow.edu.au

Follow this and additional works at: https://ro.uow.edu.au/eispapers

Part of the Engineering Commons, and the Science and Technology Studies Commons

Research Online is the open access institutional repository for the University of Wollongong. For further information contact the UOW Library: research-pubs@uow.edu.au 


\title{
Intelligent control of electroactive polymer actuators based on fuzzy and neurofuzzy methodologies
}

\begin{abstract}
Conjugated conducting polymer actuators, especially those based on polypyrrole (PPy), possess enormous potential for the creation of biomimetic devices, single-cell manipulators, numerous biomedical applications as well as robotics and prosthetics. This is due to their low actuation voltage, ability to operate at the macro- or microscale, large force-to-weight ratio, biocompatibility, low cost and their operation in aqueous and nonaqueous environments. This paper experimentally investigates the potential of intelligent control methodologies to improve the positional accuracy and response speed of trilayer PPy actuators. Two intelligent control techniques were designed and implemented - fuzzy logic PD+I control and neurofuzzy adaptive neural fuzzy inference system (ANFIS) control, which are fundamentally model-free control techniques. The performance of these controllers was compared to that of a conventional proportional integral derivative (PID) controller. It was found that the two intelligent control schemes significantly outperformed the conventional PID controller in both step and dynamic responses, with an improvement in rise time of at least 18 times and in settling time of at least two times. This study is the first to implement and compare fuzzy logic PD+I and neurofuzzy ANFIS PD+I intelligent control methodologies with a classical PID controller to the emerging field of conducting polymer PPy actuators and lays the groundwork for their use in functional devices.
\end{abstract}

\section{Keywords}

Electroactive polymer actuators, fuzzy logic, neural networks, smart actuators

\section{Disciplines}

Engineering | Science and Technology Studies

\section{Publication Details}

Druitt, C. M. \& Alici, G. (2014). Intelligent control of electroactive polymer actuators based on fuzzy and neurofuzzy methodologies. IEEE-ASME Transactions on Mechatronics, 19 (6), 1951-1962. 


\title{
Intelligent Control of Electroactive Polymer Actuators Based on Fuzzy and Neuro-Fuzzy Methodologies
}

\author{
C. M. Druitt, IEEE Member, G.Alici,
}

\begin{abstract}
Conjugated conducting polymer actuators, especially those based on polypyrrole (PPy), possess enormous potential for the creation of bio-mimetic devices, single-cell manipulators, numerous biomedical applications as well as robotics and prosthetics. This is due to their low actuation voltage, ability to operate at the macro or micro scale, large force-to-weight ratio, biocompatibility, low cost and their operation in aqueous and non-aqueous environments. This paper experimentally investigates the potential of intelligent control methodologies to improve the positional accuracy and response speed of tri-layer polypyrrole actuators. Two intelligent control techniques were designed and implemented - Fuzzy Logic PD+I control and Neuro-Fuzzy Adaptive Neural Fuzzy Inference System (ANFIS) control, which are fundamentally model-free control techniques. The performance of these controllers was compared to that of a conventional Proportional Integral Derivative (PID) controller. It was found that the two intelligent control schemes significantly outperformed the conventional PID controller in both step and dynamic response, with an improvement in rise time of at least 18 times and in settling time of at least 2 times. This study is the first to implement and compare Fuzzy Logic PD+I and Neuro-Fuzzy ANFIS PD+I intelligent control methodologies with a classical PID controller to the emerging field of conducting polymer polypyrrole actuators and lays the groundwork for their use in functional devices.
\end{abstract}

Index Terms-Smart Actuators, Fuzzy Logic, Neural Networks, Electroactive Polymer Actuators.

\section{INTRODUCTION}

$\mathrm{T}$ RI-LAYER polypyrrole actuators are an emerging technology which possesses enormous potential for future macro, micro and nano scale applications such as bio-mimetic devices, single cell manipulators, biomedical applications and prosthetics. These applications are made possible due to the unique mechanism of actuation which consists of the

Manuscript received October 20, 2013. This work was supported by the ARC Centre of Excellence for Electromaterials Science.

C. M. Druitt, was a mechatronic engineering honours undergraduate with the School of Mechanical, Materials and Mechatronic Engineering at the University of Wollongong, NSW, 2522 AUS. He is now with BAE Systems, North Ryde, NSW, 2113, AUS (e-mail: cmd096@uowmail.edu.au).

G. Alici is with the ARC Centre of Excellence for Electromaterials Science, University of Wollongong, NSW, 2522, AUS (phone 02-4221-4145; e-mail: gursel@uow.edu.au). Corresponding author: G. Alici. application of a voltage and current which is converted into mechanical output via an electrochemical redox reaction. The associated volume expansion and contraction which occurs within the polypyrrole layers causes a strain differential to occur which subsequently causes bending of the cantilevered polymer structure. As their mode of operation is similar to that of the skeletal muscle, conducting polymer actuators are also known as artificial muscles.

A PPy polymer actuator consists of five layers (Fig. 1, three of which are primary layers). The middle layer is a $110 \mu \mathrm{m}$ thick layer of poly(vinylidene fluoride) (Immobilon-P, Millipore) (PVDF) which is the backing membrane and electrolyte reservoir. The electrolyte used was $0.1 \mathrm{M}$ bis(triflouromethanesulfonyl)imide ( $\mathrm{Li}+\mathrm{TFSI}-$ ) in the solvent propylene carbonate (PC, Sigma-Aldrich). The PVDF layer is sputter coated with $10-100 \AA$ of gold on both sides for electrical conductivity. Finally, the two outermost layers consist of $30 \mu \mathrm{m}$ of galvanostatically grown PPy.

Whilst the step and dynamic responses of the polypyrrole actuators can be significantly improved through inversion based feed forward control [1], eliminating the need for a displacement sensor, this control method is heavily dependent upon a mathematical model for the system. Since the method of actuation is not completely understood and is an active area of research, eliminating the dependence upon such a model would remove a potential source of error. A non-model based feedback control method was evaluated in [2] where it was shown that it was especially resilient to drift maintaining the specified position for 20 minutes, significantly longer than [1]

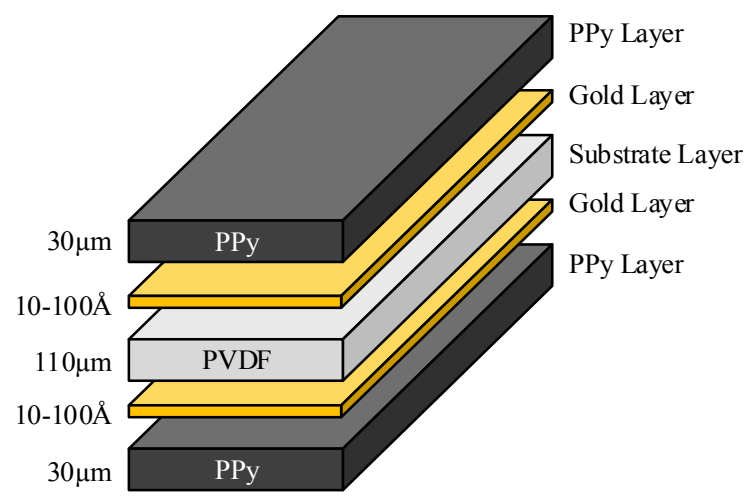

Fig. 1. Exploded view of the structure of a PPy actuator 
which recorded an increase in error over a time period of 300 seconds. A possible reason for this behaviour, as put forth in [1], was inaccuracies in the mathematical model over long time periods.

This paper aims to improve the transient response and positional accuracy of polypyrrole actuators through the application of model independent intelligent control methodologies. A preliminary version of this study has been reported in [3]. Two intelligent controllers, a Fuzzy Logic PD + I and a Neuro-Fuzzy ANFIS PD+I, are experimentally compared to a classical PID controller which serves as a performance baseline.

The primary contribution of this paper is to design and experimentally evaluate the model-free neuro-fuzzy type control strategies for conducting polymer actuators typified by tri-layer actuators considered in this study. These actuators can operate both in dry and wet media as opposed to their predecessors. This widens their application areas, provided that their positioning accuracy is enhanced through control strategies, which are robust to actuator parameter variations and external disturbances. The proposed control strategies can also be applied to other smart material actuators with similar topologies.

The organisation of this paper is as follows: Section II investigates previous control attempts, Section III presents the experimental setup and actuator synthesis, Section IV details the control theory and methodologies, Section V experimentally compares the controller performance and the final section presents the conclusions.

\section{PREVIOUS CONTROL ATtEMPTS}

The development of accurate and high performance controllers are essential for the practical application of polymer actuators. Under a constant voltage, after approximately 5 seconds, PPy polymer actuators display constant displacements which allows for simple feed-forward gain control. Whilst this allows for the determination of the input voltage to achieve a specified displacement, no improvement in dynamic response will occur.

As previously stated, an inversion based feed-forward controller was proposed that used the inverse of the plant model to counteract the dynamics in the system [1]. This model requires experimental determination of the frequency response of the actuator and, from this, the derivation of the actuator transfer function. Whilst this system displays significant improvement in rise time, delay and accuracy of the system, it suffers from excessive displacement error after 500 actuator cycles and some steady state error. The feed-forward inversion technique is not readily applicable to other samples without re-determination of the actuator's transfer function.

Further research into inversion based control methods for polymer actuators compared inversion-based ProportionalIntegral (PI) control [4] with the feed-forward inversion control previously outlined. Through the inclusion of feedback and the integral term, creep and steady state error were eliminated in the inversion PI controller whilst also demonstrating superior rise time and dynamic performance, in comparison with a standard inversion-based controller.

Clearly the inaccuracies in the actuator model can be overcome through feedback control, as demonstrated in the development of a PID based feedback controller [2]. The PID controller produced no steady state error and was able to maintain a specified displacement over a 20 minute period whilst also being able to compensate for any tip disturbance such as air resistance.

A self-tuning regulator based upon an electrochemomechanical model was developed which estimates system parameters on-line and develops a controller accordingly [5]. The effectiveness of this control structure was determined via control of PPy actuators and compared with PID and model-following control approaches. The selfupdating of model parameters by the controller made it significantly more effective in terms of tracking error than fixed model control and also PID control.

To be able to design and optimise a controller to compensate for system dynamics, a Model Reference Adaptive Control (MRAC) scheme utilising a genetic algorithm for optimisation was developed for tracking control of an IPMC type polymer actuator [6]. Whilst these actuators differ from the PPy type, the control scheme is equally applicable. Poleplacement is utilised for the implementation of the MRAC and the genetic algorithm optimises the MRAC to achieve a minimum overshoot, settling time, energy and tracking error. The controller was evaluated via simulation of the reference model, which limits the scope of the results as they cannot be directly compared to previous real-world controller performance.

Other control methodologies applied to IPMC (ionicpolymer metal composite) type actuators include adaptive neuro-fuzzy control [7] and the development of a non-linear black box model [8] which utilises the learning capability of neural networks. The neuro-fuzzy controller developed by Thinh et al [7] compares a pure fuzzy controller with an adaptive neuro-fuzzy controller and demonstrates that a neural-network is capable of modelling and improving the performance of a dynamic system. Truong et al [8] attempts to model the IPMC actuator in order to eliminate the need for an external displacement sensor. Their approach utilises a general multi-layer perceptron neural network combined with a selfadjustable learning mechanism to form the non-linear black box model. Both of these studies highlight the effectiveness of intelligent control, in particular the benefit of adding a neuralnetwork to the controller or model.

The most significant problems associated with creating an accurate, responsive control scheme for polymer actuators is the tendency of the polymer's displacement to drift over time as the dynamics of the polymer change, and the inconsistency between samples necessitating adjustment of the controller on a sample by sample basis. Therefore, the use of model based control systems such as inversion control introduces errors and inaccuracies into the system. Intelligent controllers such as those based on fuzzy logic or neural networks operate without any dependence upon system models and are thus well suited to the control of polymer actuators. Due to the effectiveness of 
learning and feedback, a neuro-fuzzy controller has been chosen to eliminate some of the problems inherent in applying classical control theory to the control of tri-layer polymer actuators. A neural network will optimise the parameters of a fuzzy controller, compensating for changes in the dynamic system. Eliminating system dynamics solves drift, and allows for application of the controller across numerous samples without the need for redesigning the controller.

\section{EXPERIMENTAL SETUP}

\section{A. Mechanism of Actuation}

The application of a potential difference to a conjugated polymer actuator causes the process of electrochemical doping to occur. Electrons flow from one PPy layer to the other and electrolyte ions flow from the PVDF layer into the oxidised PPy layer, and out of the reduced PPy layer to bring charge neutrality to the system [9-12]. Conjugation, the alternating single and double carbon-carbon bonds in the polymer backbone, results in positive charge carriers upon the removal of electrons from the polymer as a result of a positive applied potential; this is the oxidation state of the redox reaction. Anions are attracted to the PPy layer, or cations forced out of the polymer depending upon ion size, to maintain charge equilibrium [11].

The process of ionic flow causes solvent to be transported from the PVDF layer into the PPy layer. The force moving the solvent particles is analogous to osmotic pressure and therefore, the volume expansion attributed by solvent is termed osmotic expansion [13]. The osmotic expansion is closely related to the number of ions in the PPy and changes with the oxidation reduction states. The associated expansion is a significant effect and combined with ionic flow, electrostatic interactions, the length of carbon-carbon bonds and the conformational changes within the polymer cause volume change within the PPy layers and therefore an introduction of strain [9-16]. In a conjugated polymer, volume expansion will occur in the oxidation state and contract during the reduction state [11]. This strain differential between the two PPy layers causes the actuation motion in the direction of the reduced layer.

The electrochemomechanical reaction present in polymer actuators is most effective around the site of induced potential difference [10]. This effect is due to the fact that constant streams of ions flow into the PPy layers from the PVDF layer and therefore, it is more pronounced at the boundary between the PVDF PPy layers and diffuses through the depth of the polymer. This characteristic means that increasing the volume of the PPy by increasing the polymerisation time actually causes a reduction in the force and stress generation capability of the actuator [17]. This is due to the fact that only a limited amount of the PPy layer is maintaining a strong redox reaction; the extra polymer serves only to add weight and stiffness to the structure. Drop in potential along the length of the polymer also decreases the strength of the redox reaction and thus the volume change; the main function of the gold sputter coating layer is to minimise these losses.

\section{B. Actuator Synthesis}

Each tri-layer polymer actuator was synthesized using a galvanostatic electrochemical polymerization process reported in our previous publications [18, 19]. A PVDF (Immobilon-P, Millipore) membrane was sputter coated with a thin layer of gold on each side. A two electrode setup was used for the polymerization (Fig. 2), with the monomer (pyrrole) concentration, time, current density and temperature fixed at $0.1 \mathrm{M}, \quad 12 \mathrm{~h}, \quad 0.10 \mathrm{~mA} / \mathrm{cm} 2$ and $-35^{\circ} \mathrm{C}$ respectively. A galvanostat (Princeton Applied Research, model 363) was used to generate constant current, which produced $30 \mu \mathrm{m}$ of PPy on each side of the PVDF substrate. Once the polymerisation process was complete the bulk sheet of actuator was washed with acetone and then soaked and stored in $0.1 \mathrm{M}(\mathrm{Li}+\mathrm{TFSI}-)$ in PC. The edges of the bulk film were trimmed and actuator strips carefully cut to the desired size using a sharp scalpel to avoid electrical contact between the two PPy layers.

The actuator geometry is shown in Fig. 3, where the actuator free length is $10 \mathrm{~mm}$ with $3 \mathrm{~mm}$ allowed for the actuator clamp mechanism. The geometry was determined after considering the impact of PPy boundary diffusion [10], actuator stiffness [20, 21], actuator curling [22], IR losses, actuator damage and length optimization [23].

\section{Experimental Setup}

A purpose built experimental setup was commissioned to implement the control strategies for the trilayer actuator (Fig. 4). The actuator input control signal was first calculated by a computer running MATLAB Real Time Workshop and QuARC at a rate of $1 \mathrm{kHz}$.

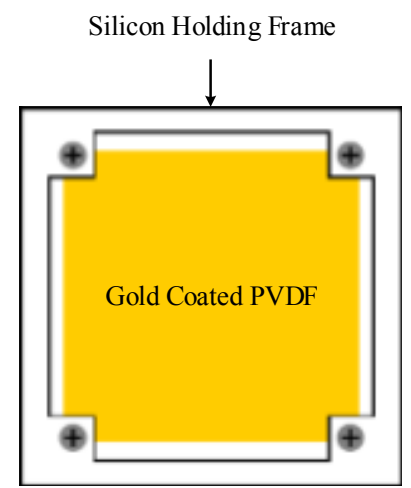

a)

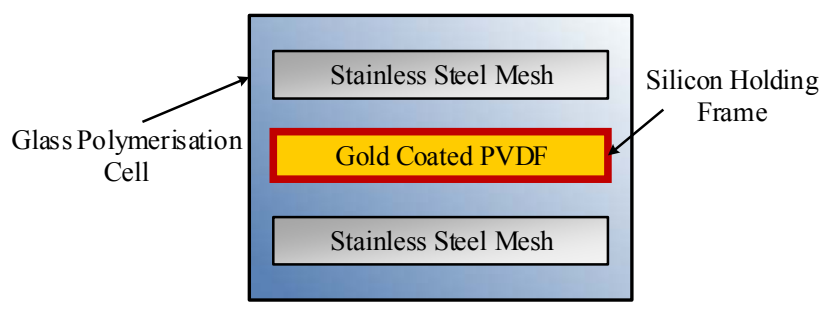

b)

Fig. 2. (a) The PVDF Holding Frame and (b) Top View of the Polymerisation Cell 
This control signal was then generated by a NI-6251 USB DAQ and output through a NI SCB-68 shielded I/O connector block. An eDAQ EA161 potentiostat operating in two electrode mode was used to amplify and apply the control signal to the actuator through an electrode clamp. An eCorder ED821 data recorder was used to record the voltage and current applied by the potentiostat at a rate of $1 \mathrm{kHz}$. Finally, a Micro-Epsilon optoNCDT 1700 Laser Displacement Sensor was used to measure the actuator tip displacement and provide feedback to the controller. The potentiostat output voltage was limited to the maximum safe operating voltage of the polpyrrole actuator, which is typically a potential difference of $2 \mathrm{~V}$ peak to peak [2].

For a valid comparison to be made, each controller is tested on the same conducting polymer actuator which was only mounted once in the electrode clamp upon the commencement of the experiment. This is to ensure that actuator free length and electrode contact resistance remain constant. For all tests, the laser was aimed $1 \mathrm{~mm}$ from the tip of the free end of the actuator. In an effort to eliminate any environmental variables such as temperature and humidity, all of the experimentation completed in this paper was performed during the same day.
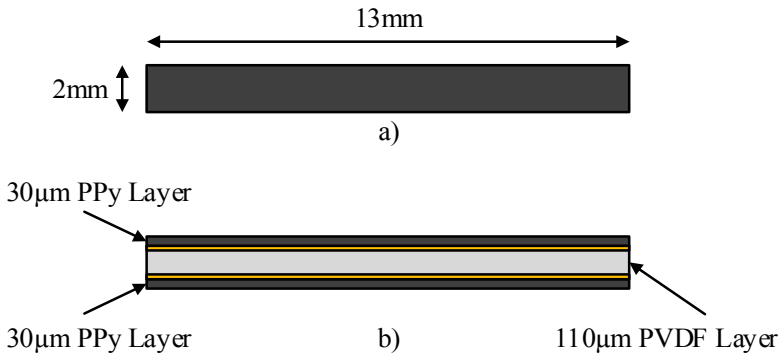

Fig. 3. Actuator Size a) Top View b) Side View

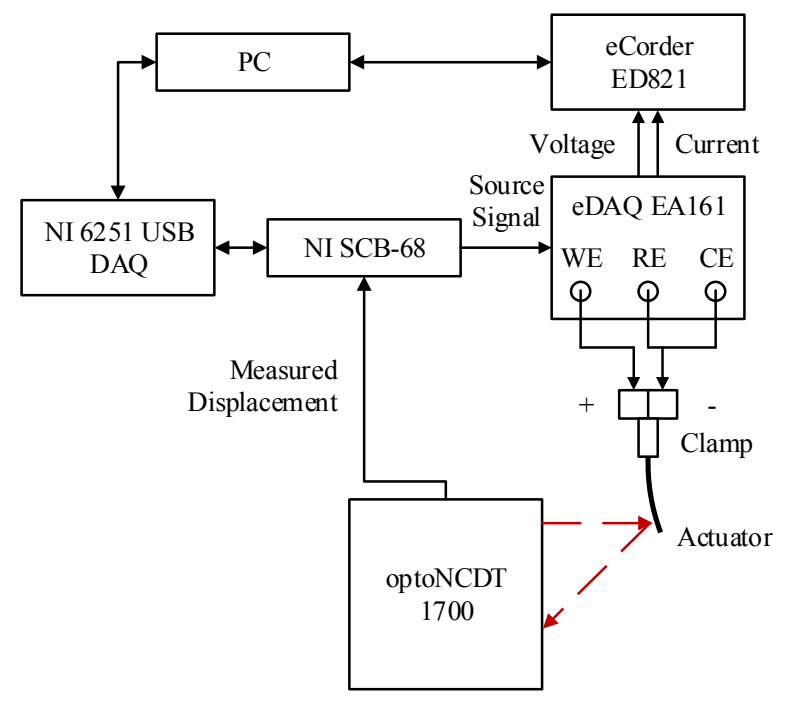

Fig. 4. Closed Loop Experimental Setup

\section{Control Methodologies}

\section{A. PID}

A Proportional Integral Derivative (PID) controller (Fig. $5(a)$ ) is considered to serve as a performance baseline to which the other two controllers are to be compared. In a previous attempt at implementing a PID controller, it was reported that the Ziegler-Nichols tuning rules resulted in PID gains which far exceeded the safe operating voltage of the polymer actuator [2]. In order to solve this problem, an Internal Model Control (IMC) PID controller (1) is used for this study tuned with simplified IMC tuning rules shown in Table I which were developed by Fruehauf [24] where L represents the dead time, $\mathrm{R}$ the slope of the response curve and $\tau$ the time constant.

To further reduce the complexity of the controller, it was suggested by Fruehauf that the derivative and filtering components (in the form of a low pass filter) were essentially the mathematical inverse of each other. Therefore, only one of these terms needs to be used at a time; otherwise derivative action and filtering action would be negating the effect of each other.

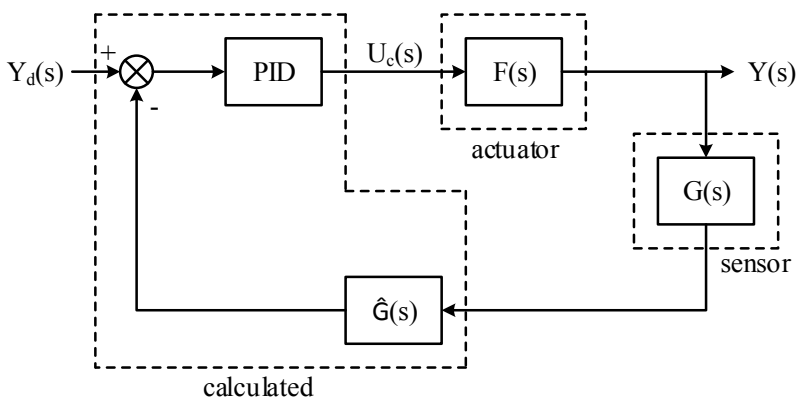

a)

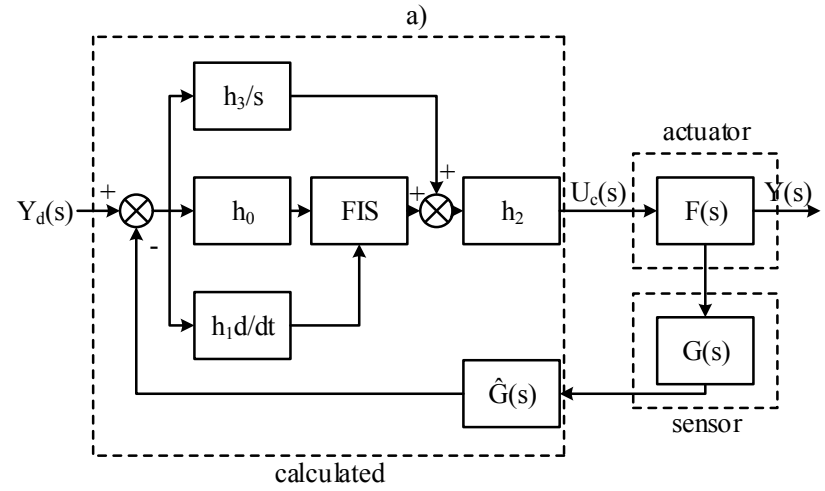

b)

Fig. 5. (a) Conventional PID Controller, (b) Intelligent PD+I Controller.

TABLE I

IMC PID TUNING RULES

\begin{tabular}{|c|c|c|c|}
\hline $\begin{array}{l}\text { IMC } \\
\text { Para. }\end{array}$ & $\tau / L>3$ & $\tau / \mathrm{L}<3$ & $\mathrm{~L}<0.5$ \\
\hline $\mathrm{K}_{\mathrm{c}}$ & $1 / 2 \mathrm{RL}$ & $1 / 2 \mathrm{RL}$ & $1 / \mathrm{R}$ \\
\hline$\tau_{\mathrm{I}}$ & $5 \mathrm{~L}$ & $\tau$ & 4 \\
\hline
\end{tabular}

$$
U_{c}(s)=K_{c}\left(1+\frac{1}{\tau_{I} s}+\tau_{D} s\right)
$$


The simplified IMC tuning rules are more robust than Ziegler-Nichols or Coohen-Coon rules. This is due to the fact that these two rules have a more aggressive tuning criterion (i.e. emphasising performance at the expense of robustness) than IMC and therefore the IMC tuning parameters do not need to be set as accurately, an adequate IMC controller can be developed from parameters within $20 \%$ of their exact value. The advantage of this flexibility is that not only would it be easier to obtain a functioning controller, but theoretically the controller should be able to also achieve satisfactory performance of numerous actuator samples not just the sample used throughout controller tuning.

To obtain the parameters necessary for the implementation of the simplified IMC tuning rules (the dead time L, the slope $\mathrm{R}$ and the time constant $\tau$ ), the open loop unit step response of the actuation process was required to obtain the displacement reaction curve. The resulting PID controller was then manually fine-tuned, as shown in (2). Due to manual tuning, this PID controller does not possess the aforementioned $20 \%$ tolerance in controller gains.

$$
U_{c}(s)=1.5+0.4 \frac{1}{s}+0.008 s
$$

\section{B. Fuzzy $P D+I$}

The most significant problems associated with creating an accurate, responsive control scheme for polymer actuators is the tendency of the polymer's displacement to drift over time as the dynamics of the polymer change, and the inconsistency between samples necessitating adjustment of the controller on a sample by sample basis. Therefore, the use of classical model based control systems can introduce errors and inaccuracies into the system $[1,4]$. Intelligent controllers such as those based on fuzzy logic or neural networks operate without any dependence upon system models and are thus well suited to the control of polymer actuators $[2,5,6]$.

Unlike a conventional PID controller, the output of the integrated error is summed separately to the proportional and derivative term. The integral term is not an input to the FIS, instead the output of the FIS is summed with the output of the integral term it is this characteristic which makes this a $\mathrm{PD}+\mathrm{I}$ controller. To develop the Fuzzy PD+I controller (Fig. 5(b)), a Fuzzy Inference System (FIS) had to be created. The FIS developed for this intelligent controller is of the TakagiSugeno-Kang (TSK) type [25, 26]. A first order TSK rule is of the form shown in (3)

$$
\begin{array}{ll}
\text { Rule } i: & \text { If } x_{1}(t) \text { is } A_{i 1}, x_{2}(t) \text { is } A_{i 2}, \ldots, \text { and } x_{n}(t) \text { is } A_{\text {in }} \\
& \text { Then } f_{i}=c_{i 0}+c_{i 1} x_{1}(t)+c_{i 2} x_{2}(t)+\ldots+c_{i n} x_{n}(t)
\end{array}
$$

where $x_{1}, x_{2}, \ldots, x_{n}$ are the antecedent input variables and $f_{i}$ is the consequent output variable. $A_{i 1}, A_{i 2}, \ldots, A_{i n}$ are the fuzzy sets defined over respective domains of $x_{1}, x_{2}, \ldots, x_{n}$ while $c_{i 0}$, $c_{i 1}, \ldots, c_{i n}$ are constant coefficients that characterise the linear relationship defined by the $i^{\text {th }}$ rule in the rule set $i=1,2, \ldots, R$. The output of a first order TSK fuzzy inference system is determined in four steps:

\section{Step 1 - Fuzzification of input variables}

Perform fuzzification of crisp input variables using a membership function. In this case a Gaussian membership function (4) was used

$$
\mu_{A_{i}}(x)=\exp \left(\frac{\left(c_{i}-x\right)^{2}}{2 \sigma_{i}^{2}}\right)
$$

where the premise parameters $c_{i}$ and $\sigma_{i}$ are the centre and width of the $i^{\text {th }}$ fuzzy set $A_{i}$ and $x$ is the crisp input. For this study, three Gaussian Membership Functions (MFs) for each of the two inputs (error and change in error) were used. These MFs were selected based upon a combination of the authors' experience and experimentation, with three membership functions for each input being used to achieve the desired controller characteristics.

\section{Step 2 - Apply fuzzy operators}

Combine antecedent input variables $x_{1}, x_{2}, \ldots, x_{n}$ to determine the firing strength (rule weight) using a T-norm product operator for AND (5) and S-norm probabilistic OR method operator for OR (6).

$$
\begin{gathered}
T_{\text {prod }}(a, b)=a b \\
S_{\text {probor }}=a+b-a b
\end{gathered}
$$

\section{Step 3 - Apply Implication method}

Determine the implication of the antecedent parameter(s) on the consequent(s) using the T-norm MIN method (7).

$$
T_{\min }(a, b)=\min (a, b)
$$

\section{Step 4 - Defuzzification}

Combine the output of all rules by calculating the weighted average (8)

$$
f=\frac{\sum_{i=1}^{n} w_{i} f_{i}}{\sum_{i=1}^{n} w_{i}}
$$

where $f$ is the crisp output, $w_{i}$ is the weight and $f_{i}$ a crisp polynomial function containing the consequent parameters.

The output of the FIS is defined by five linear functions governed by nine control rules. The control surface of this FIS is shown in Fig. 6. Once the FIS was developed, manual controller fine-tuning was undertaken. This procedure is similar to that used for the conventional PID controller. The resulting Fuzzy PD+I controller gains are shown in Table II.

\section{ANFIS}

Implementation of the Adaptive Neuro Fuzzy Inference System (ANFIS) PD+I controller (Fig. 5(b) where the FIS is replaced by an ANFIS) required an ANFIS to be created. An ANFIS is a type of hybrid neuro-fuzzy system where a neural network is functionally equivalent to a fuzzy inference system $[27,28]$. This neural network is trained to develop IF-THEN fuzzy rules and determine membership functions for input and output system variables. The structure of a hybrid neuro-fuzzy 


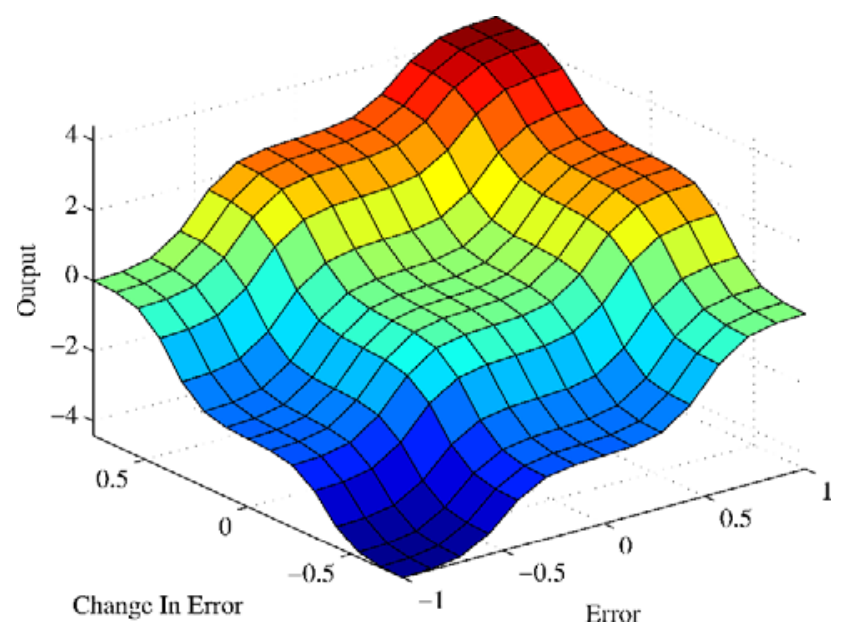

Fig. 6. Fuzzy PD+I Control Surface

TABLE II

FUZZY PD+I CONTROLLER GAINS

\begin{tabular}{cc}
\hline Gain & Value \\
\hline $\mathrm{H}_{0}$ & 2.5 \\
$\mathrm{H}_{1}$ & 0.005 \\
$\mathrm{H}_{2}$ & 2.6 \\
$\mathrm{H}_{3}$ & 0.6 \\
\hline
\end{tabular}

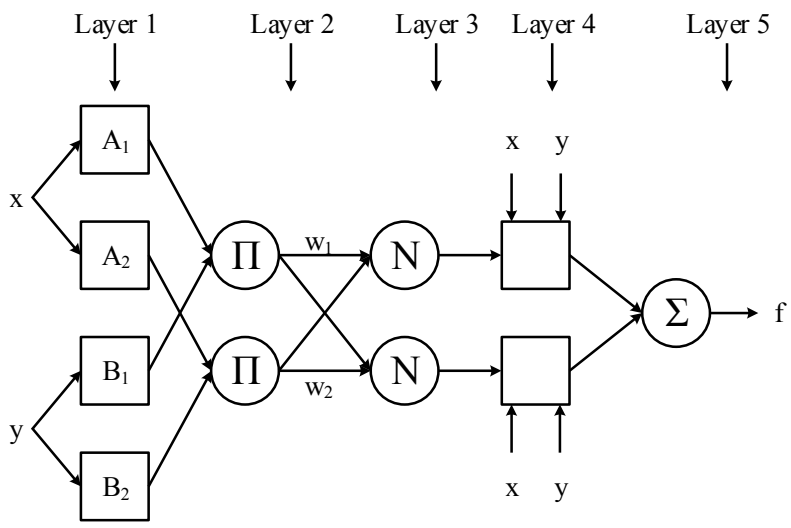

Fig. 7. Example ANFIS structure with 2 inputs and 5 layers.

system is similar to a multi-layer neural network. Typically the hybrid system has an input and output layer as well as multiple hidden layers which represent the fuzzy membership functions and rules. The structure of an example 2 input 5 layer ANFIS is shown in Fig. 7.

As per a FIS, an ANFIS contains premise parameters $\left(S_{1}\right)$, consequent parameters $\left(S_{2}\right)$ and the total set of parameters $(S)$ which contains both $S_{1}$ and $S_{2}$. It can be said that, for an ANFIS, the consequent parameters are the weights and threshold values for the output layer and the premise parameters are the weights and threshold values for the hidden layer(s).

\section{Layer 1 - Fuzzification Layer}

Neurons in this layer perform fuzzification of crisp inputs. Every node $i$ is an adaptive node with membership grade of a fuzzy set. The output of layer 1 is therefore:

$$
\mathrm{O}_{1, i}=\mu_{A_{i}}(x)
$$

where $A_{i}$ is the $i^{\text {th }}$ fuzzy set and $x$ is the crisp input to the $i^{\text {th }}$ node. In this case with a Gaussian Bell membership function $\mu_{A_{1}}(x)$ is of the form shown in (10)

$$
\mu_{A_{i}}(x)=\frac{1}{1+\left|\frac{x-c_{i}}{a_{i}}\right|^{2 b_{i}}}
$$

where $b_{i}$ is positive and $c_{i}$ locates the centre of the curve. Parameters in this layer are referred to as premise (or antecedent) parameters. Thus the premise $S_{1}$ parameter set is $\left\{a_{i}, b_{i}, c_{i}\right\}$.

Layer 2 - Rule Layer

Each neuron in this layer corresponds to a single TSK type fuzzy rule. Each rule neuron receives an input from the respective fuzzification neuron and calculates the firing strength of the rule it represents. The antecedents are evaluated by the T-norm product operator (5).

An example output of the rule neuron for $i=1,2$ is:

$$
O_{2, i}=w_{i}=\mu_{A_{i}}(x) \mu_{B_{i}}(x)
$$

where $w_{i}$ represents the firing strength of the rule $i$.

Layer 3 - Normalisation Layer

Each neuron in the normalisation layer receives inputs from all other neurons in the rule layer and calculates the normalised firing strength of a given rule (12). The normalised firing strength is the ratio of the firing strength for a given rule to the sum of firing strength of all rules, thus it represents the contribution of a given fuzzy rule to the final output

$$
O_{3, i}=\overline{w_{i}}=\frac{w_{i}}{\sum_{1}^{n} w_{n}}
$$

where $\bar{w}_{i}$ represents the normalised firing strength of the $i^{\text {th }}$ rule, $w_{i}$ represents the firing strength of the $i^{\text {th }}$ rule and $n$ is the total number of rules.

\section{Layer 4 - Defuzzification Layer}

Each neuron in the defuzzification layer is connected to its respective normalisation neuron and also receives inputs from $x$ and $y$ as per Fig. 7. A defuzzification neuron calculates the weighted consequent value of a given rule as per (13)

$$
O_{4, i}=\overline{w_{i}} f_{i}=\overline{w_{i}}\left(p_{i} x_{P}+q_{i} y_{P}+r_{i}\right)
$$

where $P$ represents the number of training data pairs (i.e., an input with a corresponding output). The consequent $S_{2}$ parameter set is therefore $\left\{p_{i}, q_{i}, r_{i}\right\}$.

\section{Layer 5 - Summation Neuron}

The neurons in the final layer calculate the sum of outputs of all defuzzification neurons and produces the overall output $f$ 
(14) which is linear in consequent parameters.

$$
O_{5,1}=f=\sum_{i} \overline{w_{i}} f_{i}=\frac{\sum_{i} w_{i} f_{i}}{\sum_{i} w_{i}}
$$

The ANFIS used in this study was created using MATLAB's hybrid neural network training method, which is a combination of least square approximation and backpropagation gradient decent methods. Each training epoch is composed of a forward pass and backward pass.

\section{Forward Pass}

In the forward pass, a training set is an input to the ANFIS and neuron outputs calculated on a layer by layer basis. During this pass the consequent parameters are optimised with least square estimation whilst the antecedent parameters remain fixed. The least squares estimation and optimisation is performed on the output layer.

As per layer 5 in a standard ANFIS, the output is of the form in (14), which can be represented as $i$ first degree linear polynomials from (13) as shown in (15)

$$
O_{5,1}=f=\sum_{i} \overline{w_{i}} f_{i}=\sum_{i} \overline{w_{i}}\left(p_{i} x_{P}+q_{i} y_{P}+r_{i}\right)
$$

and subsequently converted to matrix notation (16).

$$
O_{5,1}=\left[\begin{array}{ccccccc}
\overline{w_{1}} x_{1} & \overline{w_{1}} y_{1} & \overline{w_{1}} & \ldots & \overline{w_{i}} x_{1} & \overline{w_{i}} y_{1} & \overline{w_{i}} \\
\vdots & \vdots & \vdots & \vdots & \vdots & \vdots & \vdots \\
\overline{w_{1}} x_{P} & \overline{w_{1}} y_{P} & \overline{w_{1}} & \ldots & \overline{w_{i}} x_{P} & \overline{w_{i}} y_{P} & \overline{w_{i}}
\end{array}\right]\left[\begin{array}{c}
p_{1} \\
q_{1} \\
r_{1} \\
\vdots \\
p_{i} \\
q_{i} \\
r_{i}
\end{array}\right]
$$

Thus $O_{5,1}$ is now in the form of $B=A X$, with $B$ representing the ANFIS output $O_{5,1}, A$ representing the matrix of coefficients and $X$ containing the column vector of consequent parameters. The dimensions of $A, X$ and $B$ are $P \times M, M \times 1$ and $P \times 1$ respectively where $M$ is the number of $S_{2}$ parameters.

The solution to $B=A X$ is

$$
X^{*}=\left(A^{T} A\right)^{-1} A^{T} B
$$

where $\left(A^{T} A\right)^{-1} A^{T}$ is the pseudo-inverse of $A$ if $A^{T} A$ is nonsingular. The least square estimator minimises the error $\|A X-B\|^{2}$ by approximating $X$ with $X^{*}$. In order to create a general solution valid for both the singular and non-singular cases of $A^{T} A$, a sequential least square estimation method of obtaining $X^{*}$ as per (18) can be used

$$
\left.\begin{array}{l}
X_{i+1}=X_{i}+S_{(i+1)}\left(b_{(i+1)}^{T}-a_{(i+1)}^{T} X_{i}\right) \\
S_{i+1}=S_{i}-\frac{S_{i} a_{(i+1)} a_{(i+1)}^{T} S_{i}}{1+a_{(i+1)}^{T} S_{i} a_{(i+1)}}
\end{array}\right\} \text { for } i=0,1, \cdots, P-1
$$

where matrix $A$ is represented by $a_{i}^{T}$, matix $B$ by $b_{i}^{T}, S_{i}$ is the convariance matrix and $X^{*}$ is equivalent to $X_{P}$. The initial conditions are $X_{0}=0$ and $S_{0}=\gamma I$ where $\gamma$ is a positive large number and $I$ is an $M \times M$ identity matrix.

The forward pass continues until $A$ and $B$ are calculated, the consequent parameters identified by the least squares method and the output error is determined. Due to the use of least square estimation, the parameters of $S_{2}$ are the global optimum point in the $S_{2}$ parameter space. Therefore, the search space for the subsequent gradient decent back propagation is significantly reduced which has the effect of lowering the convergence time in the backward pass.

\section{Backward Pass}

In the backward pass, the error signals obtained from the forward pass are propagated back through the neural network, and the antecedent parameters updated using gradient decent chain rule. During the backward pass the antecedent parameters are optimised and the consequent parameters are kept fixed.

Since the forward pass has initialised the neurons in the hidden layer and optimised the output layer weights, the backward pass consists only of optimising the hidden layer weights. Take for example an adaptive network with $L$ layers with the $k^{\text {th }}$ layer consisting of \# $(k)$ nodes. The node in the $i^{\text {th }}$ position in the $k^{\text {th }}$ layer is denoted by $(k, i)$ and the node function is $O_{i}^{k}$. The error measure is therefore

$$
E_{p}=\sum_{m=1}^{\#(L)}\left(T_{m, p}-O_{m, p}^{L}\right)^{2}
$$

where $T_{(m, p)}$ represents the $m^{\text {th }}$ component of the $p^{\text {th }}$ target output vector and $O_{m, p}^{L}$ represents the $m^{\text {th }}$ component of the actual output vector produced by the introduction of the $p^{\text {th }}$ input vector.

From this result the error rate for the output node $(L, i)$ can be determined from

$$
\frac{\partial E_{p}}{\partial O_{i, p}^{L}}=-2\left(T_{i, p}-O_{i, p}^{L}\right)
$$

Using the chain rule, the error rate at the internal node $(k, i)$ can be derived as shown in (21)

$$
\frac{\partial E_{p}}{\partial O_{i, p}^{k}}=\sum_{m=1}^{\#(k+1)} \frac{\partial E_{p}}{\partial O_{m, p}^{(k+1)}} \frac{\partial O_{m, p}^{(k+1)}}{\partial O_{i, p}^{k}}
$$

where $1 \leq k \leq L-1$. Now if $\alpha$ is a parameter of the given adaptive network, the internal node error rate becomes

$$
\frac{\partial E_{p}}{\partial \alpha}=\sum_{O^{*} \in S} \frac{\partial E_{p}}{\partial O^{*}} \frac{\partial O^{*}}{\partial \alpha}
$$

where $S$ is the set of nodes with outputs depending on $\alpha$. The overall error measure $E$ with respect to $\alpha$ can be expressed as

$$
\frac{\partial E}{\partial \alpha}=\sum_{p=1}^{P} \frac{\partial E_{p}}{\partial \alpha} .
$$

Therefore the update formula for the parameter $\alpha$ is 


$$
\Delta \alpha=-\eta \frac{\partial E}{\partial \alpha}
$$

which is representable of the gradient decent method. The learning rate $\eta$, can be expressed as

$$
\eta=-\frac{k}{\sqrt{\sum_{\alpha}\left(\frac{\partial E}{\partial \alpha}\right)^{2}}}
$$

where $k$ is the step size or the length of each gradient transition. Adjusting $k$ has the effect of speeding up the rate of convergence. In this study the ANFIS utilises offline learning so the update formula for $\alpha$ is based upon (23).

20 training epochs were used for the development of the ANFIS system. Several ANFIS were developed using different combinations of membership functions and training data. After manually tuning each ANFIS PD+I controller and extensive experimental evaluation, a Gaussian Bell based ANFIS system was selected (Fig. 8). This ANFIS is trained with training data consisting of FIS input and output data gathered from the Fuzzy PD+I controller performance tests which resulted in 350,000 data points. The Gaussian Bell ANFIS PD+I controller gains are shown in Table III.

\section{Performance Characterisation}

\section{A. Step Response}

The calculated control signal and actuator tip displacement for a $1 \mathrm{~mm}, 2 \mathrm{~mm}$ and $3 \mathrm{~mm}$ step input are shown in Figs. 9 to 11 for the three control systems. The actuators used in this study are one end free, the other end fixed cantilever type

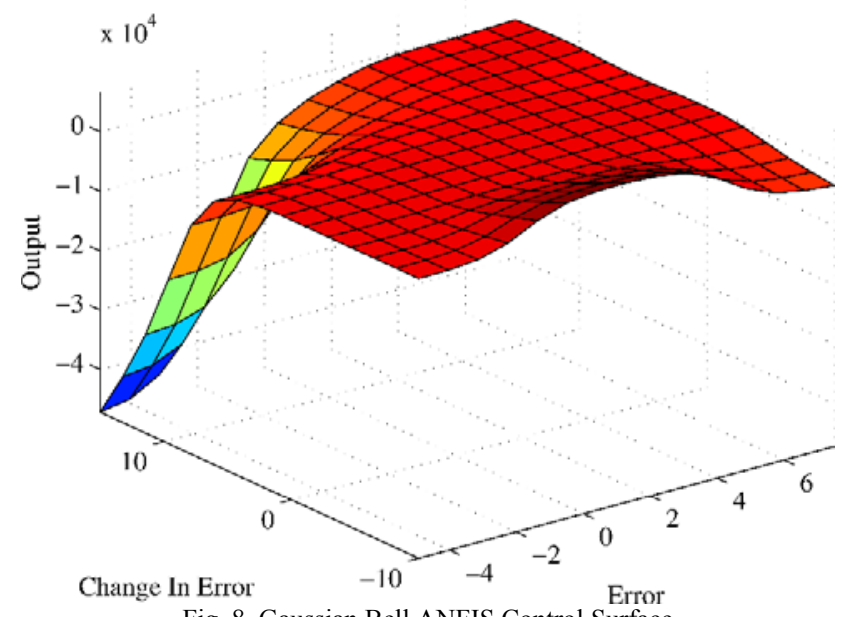
Fig. 8. Gaussian Bell ANFIS Control Surface

TABLE III

\begin{tabular}{cc} 
ANFIS CONTROLLER GAINS \\
\hline Gain & Value \\
\hline $\mathrm{H}_{0}$ & 0.4 \\
$\mathrm{H}_{1}$ & 0.0025 \\
$\mathrm{H}_{2}$ & 1.5 \\
$\mathrm{H}_{3}$ & 2.2
\end{tabular}

bending actuators with dimensions of $10 \mathrm{~mm} \times 2 \mathrm{~mm} \times 0.17$ $\mathrm{mm}$. Summarised in Tables IV to VI are the $10 \%-90 \%$ rise time, $\pm 2 \%$ settling time and percentage overshoot. For the $2 \mathrm{~mm}$ and $3 \mathrm{~mm}$ cases the results were normalised to $1 \mathrm{~mm}$. It is clearly evident that the two intelligent control approaches significantly improve upon the performance of the classical PID controller. During the $1 \mathrm{~mm}$ step response test, the intelligent controllers achieved a 119-161 fold improvement in rise time and a 2-5 fold improvement in settling time when compared to the PID performance baseline. Similarly for the $2 \mathrm{~mm}$ step response tests the intelligent control methodologies gained a performance improvement of 48-69 times for rise time and 2-8 times for settling time. Lastly, for the $3 \mathrm{~mm}$ step response test, the intelligent controllers achieved an 18-33 times improvement in rise time and a 2-20 time improvement in settling time.

From these results, it has been shown that the controllers are successfully able to compensate for the actuator dynamics under a step input. Also evident is how the controllers apply an initial high amplitude voltage spike at the beginning of each applied input signal. The purpose of this is to rapidly move the actuator to near the desired set point, the effect of which is a fast transient response. After this initial spike, the voltage decreases to a relatively constant value which remains during the entire steady state phase of displacement response, the purpose of which is to keep the actuator at the steady state position. This is due to the fact that a constant voltage means
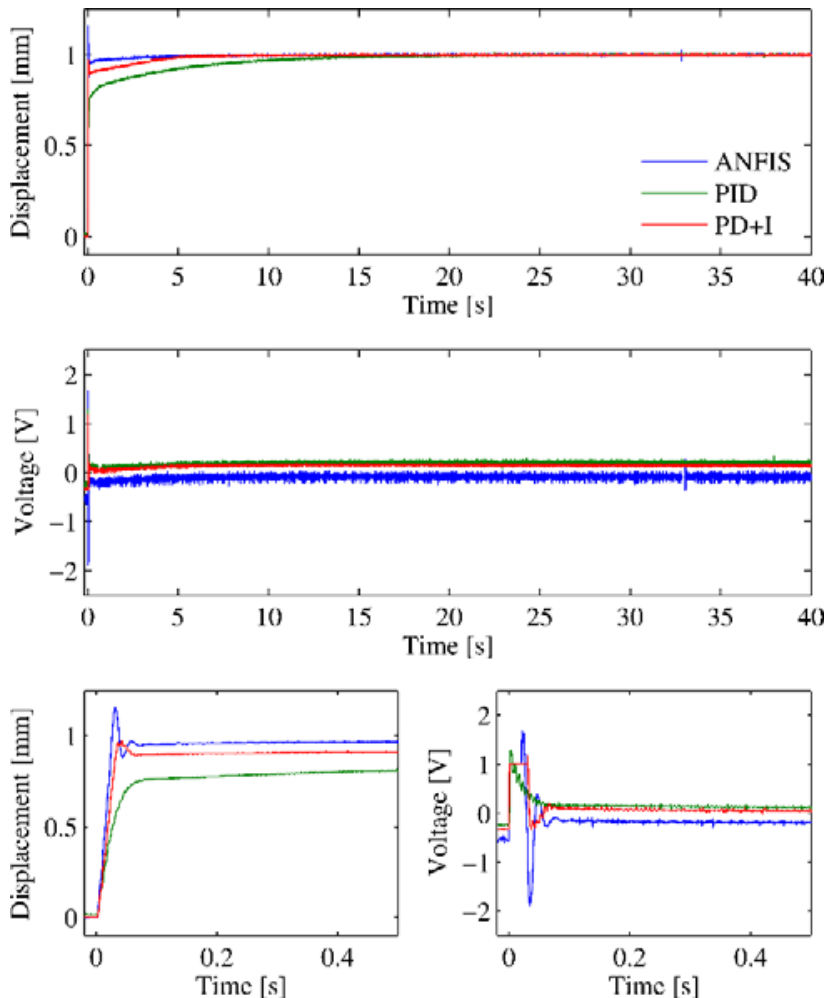

Fig. 9 Actuator displacement and input voltage of a $10 \mathrm{~mm}$ long, $2 \mathrm{~mm}$ wide polypyrrole actuator under PID, Fuzzy PD+I and ANFIS control in response to a $1 \mathrm{~mm}$ step input. The bottom two plots show the first 0.5 seconds of the displacement response and the control voltage. 

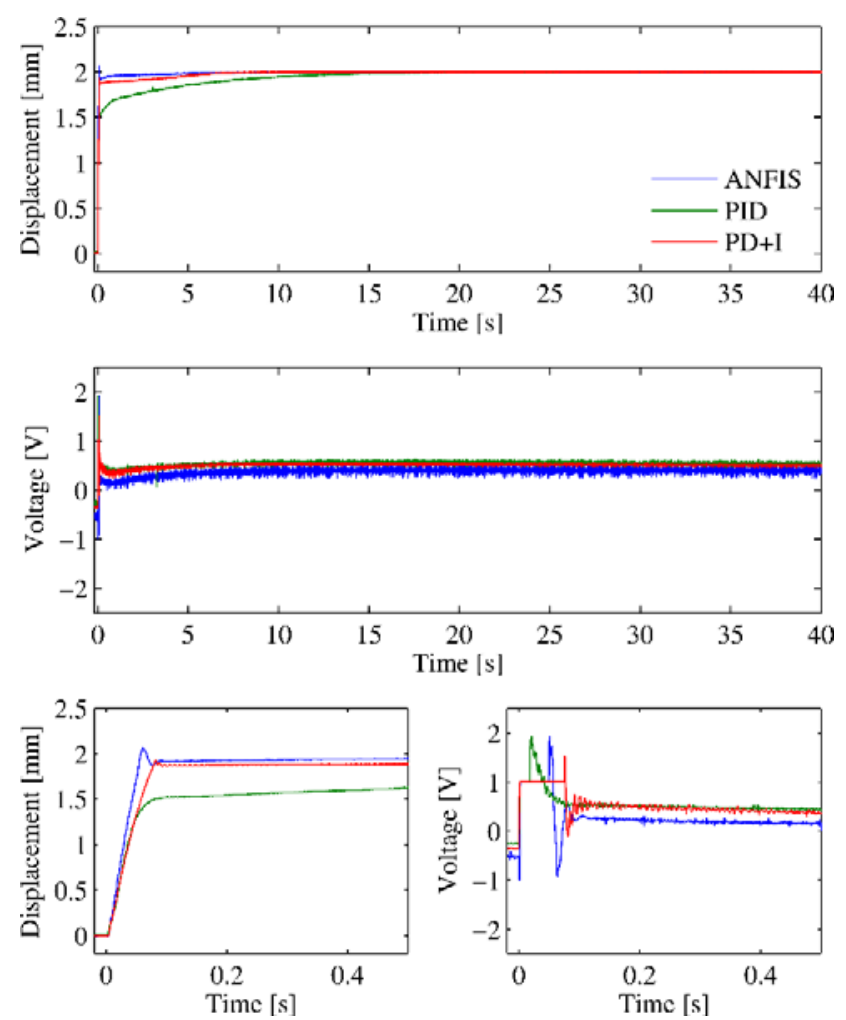

Fig. 10 Actuator displacement and input voltage of a $10 \mathrm{~mm}$ long, $2 \mathrm{~mm}$ wide polypyrrole actuator under PID, Fuzzy PD + I and ANFIS control in response to a $2 \mathrm{~mm}$ step input. The bottom two plots show the first 0.5 seconds of the displacement response and the control voltage
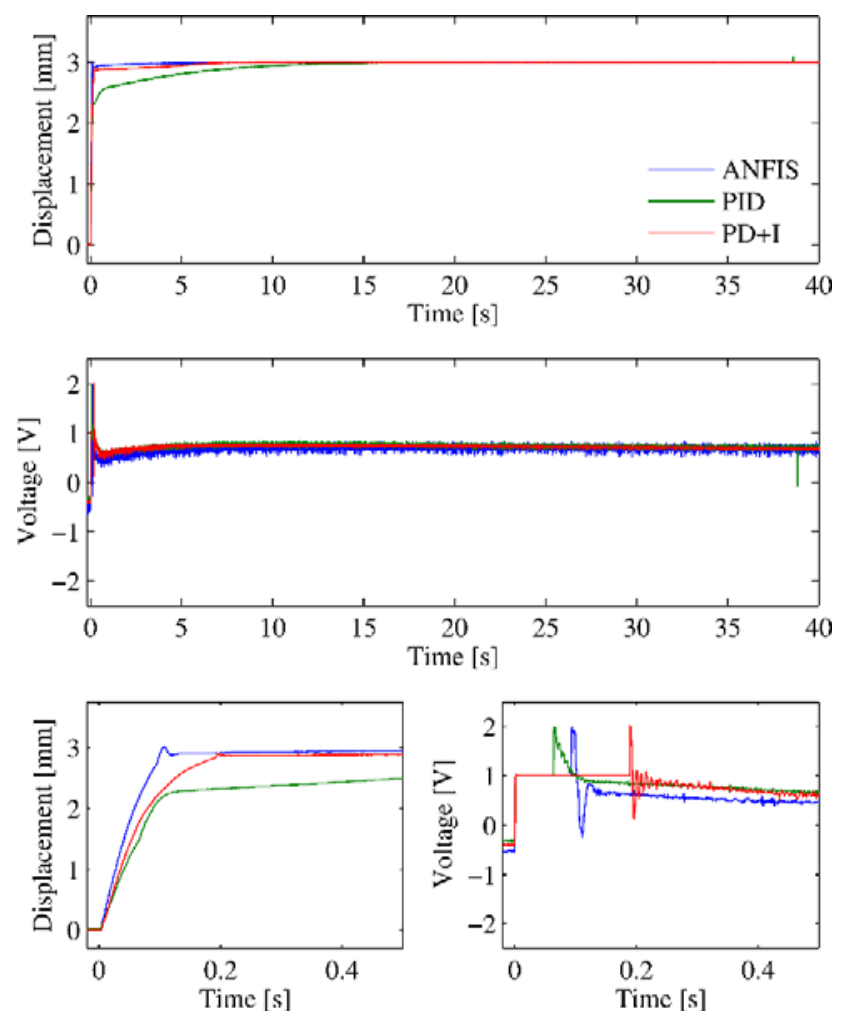

Fig. 11 Actuator displacement and input voltage of a $10 \mathrm{~mm}$ long, $2 \mathrm{~mm}$ wide polypyrrole actuator under PID, Fuzzy PD+I and ANFIS control in response to a $3 \mathrm{~mm}$ step input. The bottom two plots show the first 0.5 seconds of the displacement response and the control voltage
TABLE IV

$1 \mathrm{~mm}$ CONTROLled STEP RESPONSE PERFORMANCE

\begin{tabular}{cccc}
\hline $\begin{array}{c}\text { Control } \\
\text { Method }\end{array}$ & $\begin{array}{c}\text { Rise Time } \\
{[\mathrm{s}]}\end{array}$ & $\begin{array}{c}\text { Settling Time } \\
{[\mathrm{s}]}\end{array}$ & $\begin{array}{c}\text { Overshoot } \\
{[\%]}\end{array}$ \\
\hline ANFIS & 0.02 & 2.068 & 15.6478 \\
PD+I & 0.027 & 4.543 & 0 \\
PID & 3.222 & 12.206 & 0 \\
\hline \hline
\end{tabular}

$2 \mathrm{~mm}$ Normalised CONTROLLED STEP RESPONSE PERFormanCE

\begin{tabular}{cccc}
\hline $\begin{array}{c}\text { Control } \\
\text { Method }\end{array}$ & $\begin{array}{c}\text { Rise Time } \\
{[\mathrm{s}]}\end{array}$ & $\begin{array}{c}\text { Settling Time } \\
{[\mathrm{s}]}\end{array}$ & $\begin{array}{c}\text { Overshoot } \\
{[\%]}\end{array}$ \\
\hline ANFIS & 0.044 & 1.264 & 3.1835 \\
PD+I & 0.063 & 5.127 & 0 \\
PID & 3.032 & 11.354 & 0 \\
\hline \hline
\end{tabular}

TABLE VI

3mm Normalised CONTROLLED STEP RESPONSE PERFormanCE

\begin{tabular}{ccc}
\hline \hline $\begin{array}{c}\text { Control } \\
\text { Method }\end{array}$ & $\begin{array}{c}\text { Rise Time } \\
{[\mathrm{s}]}\end{array}$ & $\begin{array}{c}\text { Settling Time } \\
{[\mathrm{s}]}\end{array}$ \\
\hline ANFIS & 0.083 & 0.486 \\
PD+I & 0.147 & 4.765 \\
PID & 2.77 & 9.999 \\
\hline \hline
\end{tabular}

that the polypyrrole layer is maintaining a constant volume and therefore, a constant tip displacement occurs.

It is also evident that the PID controller rise time and settling time actually decreases when the set point is raised. This shows that the system is becoming less damped as the set-point is raised, which could mean that the PID controller will become unstable as the set point is raised past $3 \mathrm{~mm}$. This was an unexpected result since it was assumed that the further the actuator was made to displace, the slower the response would be. By analysing the controller output data, it could be theorized that the speed of actuator displacement response is directly proportional to actuator input voltage since this voltage increases with an increase in displacement set point. This corresponds to the actuator displacement mechanism of volume expansion, as an increase in actuator input voltage causes a stronger redox reaction to occur. Therefore, a larger volume change takes place within the polypyrrole and evidently the speed of this reaction also increases.

The two intelligent controllers, however, do not display this characteristic of a reduction in settling time and rise time with an increase in setpoint. The Fuzzy PD+I controller demonstrates an increase in rise time and a relatively steady settling time. Whereas the Neuro Fuzzy ANFIS controller exhibits an increase in rise time, a decrease in settling time and a reduction in overshoot from around $\% 15$ in the $1 \mathrm{~mm}$ step down to zero in the $3 \mathrm{~mm}$ step. By analysing the actuator input voltage plots for the two intelligent controllers, it would appear that the duration of the initial high voltage spike plays a significant role in the transient response of the actuator. This occurs since an increase in duration of the voltage spike would allow more time for the ions move in and out of the two PPy layers. Therefore a faster speed of response and a much smaller tracking error are obtained. 


\section{B. Dynamic Response}

The tracking performance of the polypyrrole actuator in response to the summed sinusoid $Y_{d}(s)=0.5 \sin (\pi t)+0.5 \sin (0.1 \pi t) \quad$ is shown in Fig. 12 for the three control approaches. The Normalised Root Mean Square (NRMS) error position tracking error for all three controllers in response to a summed sinusoid and a square wave at four different frequencies is shown in Table VII. If the error is given by $x=Y_{d}(s)-\hat{G}(s)$, the NRMS error will be

$$
N R M S=\underbrace{\sqrt{\frac{1}{n} \sum_{i=1}^{n}\left(x_{i}\right)^{2}}}_{\text {RMS }} \times \underbrace{\frac{100}{x_{\max }-x_{\min }}}_{\text {Normalise } \%}
$$

where $n$ is the number of data points.

All controllers are able to track the summed sinusoid with acceptable error levels. However, the error rates in the square wave frequency response tests are significantly higher. It can clearly be seen from the testing of summed sinusoidal tracking that the two intelligent controllers again significantly outperform the conventional PID system. The Fuzzy PD+I controller demonstrates a 1.92 times improvement in sinusoidal positional accuracy compared to the PID controller. However, the PD $+\mathrm{I}$ controller is shown to become unstable at frequencies above $1 \mathrm{~Hz}$, which shows that the controller is unable to compensate quickly enough to the actuator. This could be overcome by retuning the controller gains, however this may have a detrimental effect on step response performance. The ANFIS controller achieves a 4.26 fold increase in sinusoidal tracking accuracy over the PID controller resulting in the ANFIS controller being the overall performance leader in the square wave frequency tests. Interestingly the PID controller outperforms the ANFIS controller in the $2 \mathrm{~Hz}$ test.

The performance of these controllers can be compared to the self-tuning regulator as reported in the literature [5] for a similar actuator topology and controller setpoint. The RMS error of the controllers from this study and the self-tuning regulator are shown in Table VIII, note that the error is in RMS [mm] not NRMS [\%] as used previously. It is evident that the ANFIS controller demonstrated a 2.24 times improvement in tracking accuracy compared to that of the selftuning regulator. From this performance comparison, the selftuning regulator is comparable in tracking accuracy to that of the $\mathrm{PD}+\mathrm{I}$ controller.

\section{Actuator Drift Resilience}

In order to determine if these control schemes are able to successfully eliminate and be resilient to the effects of changing actuator dynamics, an extended duration $1 \mathrm{~mm}$ step response test was performed where the controller set point was maintained for 800 seconds and is shown in Fig. 13. This duration is around 20 times longer than the step response tests performed previously in this study, and is more than twice as long as the extended duration test performed in [1]. From Fig. 13 it is clear that all control schemes are able to compensate
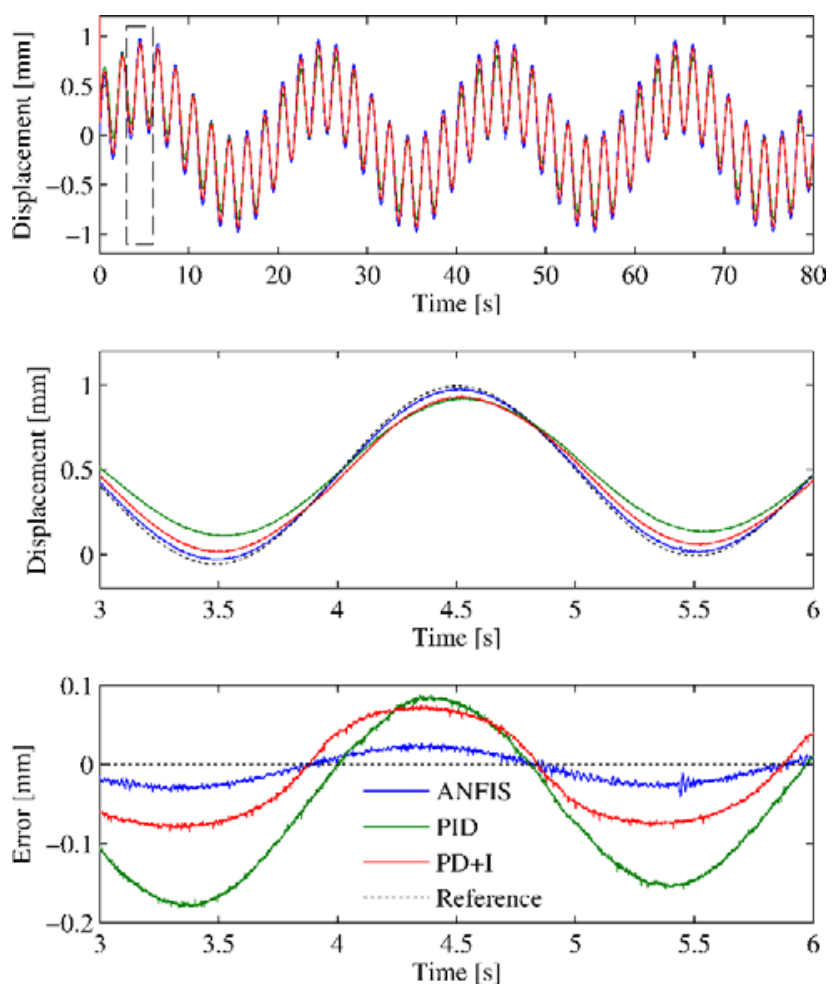

Fig. 12 Actuator displacement and tracking error of a $10 \mathrm{~mm}$ long, $2 \mathrm{~mm}$ wide polypyrrole actuator under PID, Fuzzy PD $+\mathrm{I}$ and ANFIS control in response to a $0.5 \sin (\pi \mathrm{t})+0.5 \sin (0.1 \pi \mathrm{t})$ sinusoid. The bottom two plots show an enlarged 3 second segment.

TABLE VII

Normalised Percentage RoOT MEAN SQUARE Position Tracking ERror

\begin{tabular}{cccccc}
\hline \hline $\begin{array}{c}\text { Control } \\
\text { Method }\end{array}$ & $\begin{array}{c}\text { Summed Sinusoid } \\
{[\%]}\end{array}$ & $\begin{array}{c}0.5 \mathrm{~Hz} \\
{[\%]}\end{array}$ & $\begin{array}{c}1 \mathrm{~Hz} \\
{[\%]}\end{array}$ & $\begin{array}{c}2 \mathrm{~Hz} \\
{[\%]}\end{array}$ & $\begin{array}{c}4 \mathrm{~Hz} \\
{[\%]}\end{array}$ \\
\hline ANFIS & 1.2498 & 14.2403 & 19.3426 & 25.9615 & 34.0353 \\
PD+I & 2.7742 & 16.5266 & 22.1817 & Unstable & Unstable \\
PID & 5.3255 & 20.4641 & 25.9313 & 21.6886 & 42.9576 \\
\hline \hline
\end{tabular}

TABLE VIII

RoOT MEAN SQUare POSITION TRACKING ERROR

\begin{tabular}{ccc}
\hline \hline $\begin{array}{c}\text { Control } \\
\text { Method }\end{array}$ & Set Point & $\begin{array}{c}\text { RMS Error } \\
{[\mathrm{mm}]}\end{array}$ \\
\hline ANFIS & $0.5 \sin (\pi t)+0.5 \sin (0.1 \pi t)$ & 0.0299 \\
PD+I & $0.5 \sin (\pi t)+0.5 \sin (0.1 \pi t)$ & 0.0611 \\
PID & $0.5 \sin (\pi t)+0.5 \sin (0.1 \pi t)$ & 0.1077 \\
$\begin{array}{c}\text { Self-Tuning } \\
\text { Regulator [5] }\end{array}$ & $0.5 \sin (\pi t)+0.5 \sin (0.2 \pi t)$ & 0.067 \\
\hline \hline
\end{tabular}

for actuator drift, this is a consequence of feedback control being used rather than the method of control used.

\section{Controller Robustness}

To establish the robustness of the controllers developed in this study, the three controllers were applied to a second new actuator sample cut from the same bulk actuator sheet with the same geometric specifications. The testing methodology applied is identical to that previously detailed for the original sample so as to facilitate a performance and characterisation comparison. The step response performance data for 1,2 and 

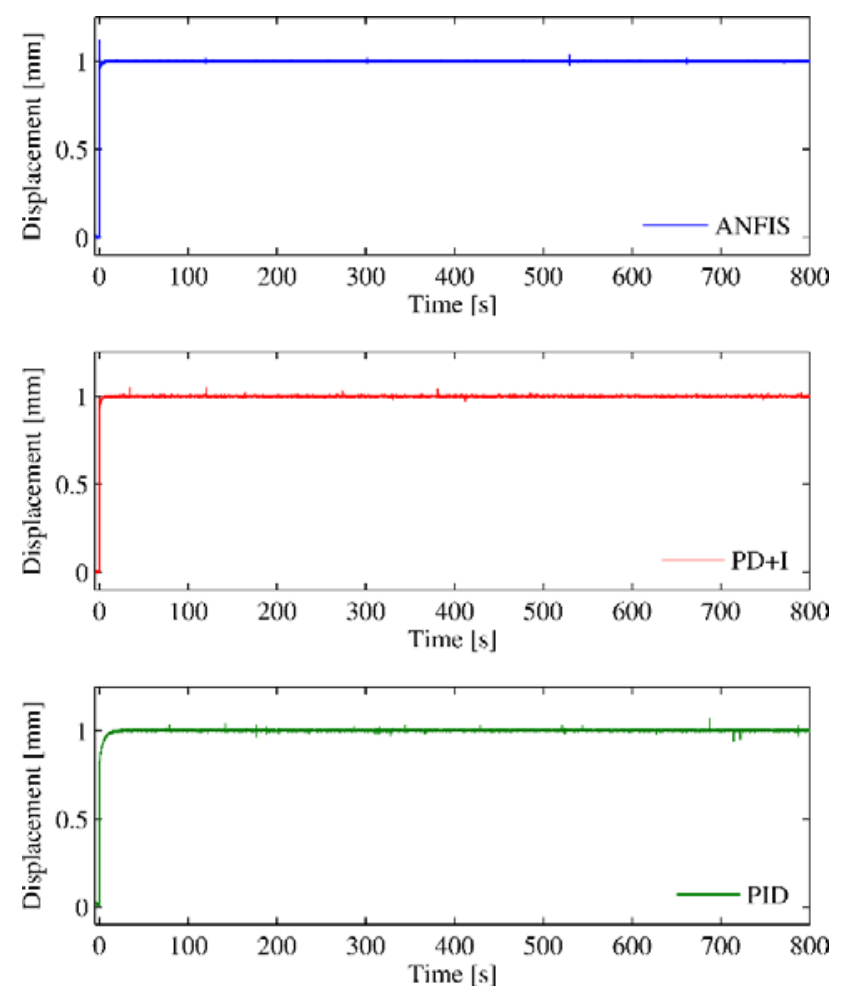

Fig. 13 Actuator extended duration displacement of a $10 \mathrm{~mm}$ long, $2 \mathrm{~mm}$ wide polypyrrole actuator under PID, Fuzzy PD+I and ANFIS control in response to a $1 \mathrm{~mm}$ step input.

$3 \mathrm{~mm}$ step displacement responses for the second actuator sample are shown in Tables IX to XI. A noticeable trend evident in these results is how the new actuator sample exhibits no overshoot. This clearly indicates different dynamics in the control system and demonstrates the variability of the actuator samples. The comparison of these step response results with those of the first actuator sample show that the second actuator is consistently slower in rise time but faster in settling time. Also, whilst the ANFIS controller maintains its performance lead in the $1 \mathrm{~mm}$ and $2 \mathrm{~mm}$ response tests, it can be unstable in the $3 \mathrm{~mm}$ test. This finding was unexpected and suggests that there can be a handful of reasons for this unstable behaviour including the variations in electrical, mechanical and chemical properties of these actuators [9]. With reference to the results in Tables IV - XI, we can still claim that the intelligent controllers are effective enough to command the smart actuators satisfactorily. Further research is needed to investigate the root cause of this unexpected behaviour under a higher setpoint. The sinusoidal and square wave tracking performance for the second actuator sample is presented in Table XII. From this data, it can be observed that the new actuator sample again demonstrates less performance than the original actuator, with the second actuator sample maintaining noticeably higher RMS tracking error throughout all tests for each controller. It is unclear whether the slower performance of the new actuator sample is caused by a variation in its geometry such as length, width, polypyrrole uniformity, the distribution of the gold clusters or electrical, mechanical and chemical properties of the actuator, which depend on the voltage applied to the actuators. This
TABLE IX

$2^{\text {ND }}$ ACTUATOR SAMPLE $1 \mathrm{~mm}$ CONTROLled Step Response PERFormance

\begin{tabular}{ccc}
\hline \hline $\begin{array}{c}\text { Control } \\
\text { Method }\end{array}$ & $\begin{array}{c}\text { Rise Time } \\
{[\mathrm{s}]}\end{array}$ & $\begin{array}{c}\text { Settling Time } \\
{[\mathrm{s}]}\end{array}$ \\
\hline ANFIS & 0.043 & 2 \\
PD+I & 0.052 & 3.829 \\
PID & 3.494 & 10.034 \\
\hline \hline
\end{tabular}

TABLE $X$

$2^{\mathrm{ND}}$ ACTUATOR SAMPLE $2 \mathrm{~mm}$ NORMALISED CONTROLled STEP RESPONSE PERFormance

Control Rise Time Settling Time

$\begin{array}{lll}\text { Method } & {[\mathrm{s}]} & {[\mathrm{s}]}\end{array}$

$\begin{array}{lll}\text { ANFIS } & 0.132 & 0.69\end{array}$

$\begin{array}{lll}\mathrm{PD}+\mathrm{I} & 0.174 & 3.72\end{array}$

$\begin{array}{lll}\text { PID } & 2.743 \quad 8.833\end{array}$

TABLE XI

$2^{\mathrm{ND}}$ ACTUATOR SAMPLE $3 \mathrm{~mm}$ NORMALISED CONTROLLED STEP RESPONSE PERFORMANCE

\begin{tabular}{ccc}
\hline Control & Rise Time \\
Method & {$[\mathrm{s}]$} & $\begin{array}{c}\text { Settling Time } \\
{[\mathrm{s}]}\end{array}$ \\
\hline ANFIS & Unstable & Unstable \\
PD+I & 0.59 & 0.995 \\
PID & 2.025 & 7.842 \\
\hline \hline
\end{tabular}

TABLE XII

$2^{\mathrm{ND}}$ ACTUATOR SAMPLE

Normalised Percentage RoOT MEAN SQUARE POSITION TRACKING ERROR

\begin{tabular}{cccccc}
\hline $\begin{array}{c}\text { Control } \\
\text { Method }\end{array}$ & $\begin{array}{c}\text { Summed Sinusoid } \\
{[\%]}\end{array}$ & $\begin{array}{c}0.5 \mathrm{~Hz} \\
{[\%]}\end{array}$ & $\begin{array}{c}1 \mathrm{~Hz} \\
{[\%]}\end{array}$ & $\begin{array}{c}2 \mathrm{~Hz} \\
{[\%]}\end{array}$ & $\begin{array}{c}4 \mathrm{~Hz} \\
{[\%]}\end{array}$ \\
\hline ANFIS & 1.5471 & 18.6083 & 24.9218 & 32.9747 & 27.6277 \\
PD+I & 3.7583 & 20.3122 & 26.9157 & 35.7291 & Unstable \\
PID & 7.8585 & 25.6733 & 32.396 & 41.809 & 53.8915 \\
\hline \hline
\end{tabular}

discrepancy could also be caused by other experimental variables such as changes in electrode contact resistance between this actuator and the original one.

It could be theorised that the established hysteresis in the original actuator is the reason for the discrepancy in actuator performance, and that continued use would eventually cause the performance of the second actuator sample to improve as the hysteresis effect establishes itself. However, it was reported by Huynh [29] that the hysteresis effect is negligibly small for a $1 \mathrm{~mm} \times 5 \mathrm{~mm}$ PPy actuator driving a rigid link. Whilst the circumstances and physical dimension are different between Huynh's evaluation and this study, it is hard to imagine such a large increase in the hysteresis effect due to changes in physical dimensions.

An alternate theory is that the structural rigidity of the polymer actuator reduces slightly over a period of use. This would explain why the original actuator demonstrates superior performance as it has been used through tuning and testing of three controllers, and why the second new actuator demonstrates a slower performance. If this theory holds, the lack of performance in the second actuator would be due to heightened rigidity in its fresh state and that continued use 
would see its performance improve. Testing of this theory is beyond the scope of this study and cannot be inferred from the results obtained herein. This is due to the fact that even the first step response tests were obtained only after extensive tuning and open loop testing.

\section{CONCLUSION}

We successfully designed and implemented two intelligent control strategies and compared their performance with that of a conventional PID controller. The factors responsible for the performance of these controllers are investigated by analysing the controller voltage output. It has been theorised that the speed of the actuators' displacement response is directly proportional to the actuators input voltage as this was found to increase with an increase in the controller setpoint. This theory relates directly to the mechanism of volume expansion where an increase in actuator input voltage causes a stronger redox to occur and therefore, a larger volume change takes place within the PPy layers.

The performance of this conventional PID controller was then compared to a fuzzy logic PD+I controller. This intelligent controller is found to demonstrate a significant improvement in settling time and tracking accuracy over the PID controller. This improvement in performance is postulated to be a result of the controllers' manipulation of the duration of applied actuator voltage, rather than operating through amplitude modification like found in the PID case. Whilst this intelligent controller demonstrated improved performance compared to the classical controller, it was found that this $\mathrm{PD}+\mathrm{I}$ controller suffers from instability at frequencies of $4 \mathrm{~Hz}$ or greater. This result placed restrictions on the implementation of this controller and limits its use to low frequency applications. Finally, a neuro-fuzzy ANFIS was developed and used to replace the FIS in the PD+I controller. It was found to significantly outperform the classical PID and the fuzzy PD+I controller developed previously.

This study has performed a rigorous experimental evaluation of the classical PID and intelligent fuzzy and neurofuzzy controllers for PPy-based electroactive polymer actuators, and sets the groundwork for the application of these actuators in functional devices.

\section{REFERENCES}

[1] S. W. John, G. Alici, and C. D. Cook, "Inversion-based feedforward control of polypyrrole trilayer bender actuators," IEEE/ASME Transactions on Mechatronics, vol. 15, pp. 149-156, 2010.

[2] Q. Yao, G. Alici, and G. M. Spinks, "Feedback control of tri-layer polymer actuators to improve their positioning ability and speed of response," Sensors and Actuators, A: vol. 144, pp. 176-184, 2008.

[3] C. D. Druitt and G. Alici, "Neuro-Fuzzy Control of Electroactive Polymer Actuators," in 2013 IEEE/ASME International Conference on Advanced Intelligent Mechatronics, AIM 2013, July 9-12, 2013, Wollongong, Australia, 2013.

[4] S. W. John, G. Alici, and C. D. Cook, "Towards the position control of conducting polymer trilayer bending actuators with integrated feedback sensor," in 2009 IEEE/ASME International Conference on Advanced Intelligent Mechatronics, AIM 2009, July 14, 2009 - July 17, 2009, Singapore, Singapore, 2009, pp. 65-70.

[5] Y. Fang, X. Tan, and G. Alici, "Robust adaptive control of conjugated polymer actuators," IEEE Transactions on Control Systems Technology, vol. 16, pp. 600-612, 2008.
[6] B. C. Lavu, M. P. Schoen, and A. Mahajan, "Adaptive intelligent control of ionic polymer-metal composites," Smart Materials and Structures, vol. 14, pp. 466-474, 2005.

[7] N. T. Thinh, Y. S. Yang, and I. K. Oh, "Adaptive neuro-fuzzy control of ionic polymer metal composite actuators," Smart Materials \& Structures, vol. 18, Jun 2009.

[8] D. Q. Truong and K. K. Ahn, "Design and Verification of a Non-linear Black-Box Model for Ionic Polymer Metal Composite Actuators," J. of Int. Material Systems and Structures, vol. 22, pp. 253-269, Feb 2011.

[9] G. Alici, B. Mui, and C. Cook, "Bending modeling and its experimental verification for conducting polymer actuators dedicated to manipulation applications", Sensors and Actuators, A, vol. 126, pp. 396-404, 2006.

[10] G. Alici, P. Metz, and G. M. Spinks, "A methodology towards geometry optimization of high performance polypyrrole (PPy) actuators," Smart Materials and Structures, vol. 15, pp. 243-252, 2006.

[11] Y. Fang, X. Tan, Y. Shen, N. Xi, and G. Alici, "A scalable model for trilayer conjugated polymer actuators and its experimental validation," Materials Science and Engineering C, vol. 28, pp. 421-428, 2008.

[12] X. Wang, B. Shapiro, and E. Smela, "Visualizing ion currents in conjugated polymers," Advanced Materials, vol. 16, pp. 1605-1609, 2004.

[13] L. Bay, T. Jacobsen, S. Skaarup, and K. West, "Mechanism of actuation in conducting polymers: Osmotic expansion," Journal of Physical Chemistry B, vol. 105, pp. 8492-8497, 2001.

[14] J. D. W. Madden, P. G. A. Madden, and I. W. Hunter, "Conducting polymer actuators as engineering materials," in Smart Structures and Materials 2002: Electroactive Polymer Actuators and Devices (EAPAD), March 18, 2002 - March 21, 2002, San Diego, CA, United states, 2002, pp. 176-190.

[15] E. Smela, "Conjugated polymer actuators for biomedical applications," Advanced Materials, vol. 15, pp. 481-494, 2003.

[16] M. R. Gandhi, P. Murray, G. M. Spinks, and G. G. Wallace, "Mechanism of electromechanical actuation in polypyrrole," Synthetic Metals, vol. 73, pp. 247-256, 1995.

[17] A. S. Hutchison, T. W. Lewis, S. E. Moulton, G. M. Spinks, and G. G. Wallace, "Development of polypyrrole-based electromechanical actuators," Synthetic Metals, vol. 113, pp. 121-127, 2000.

[18] S. John, G. Alici, and C. Cook, "Frequency response of polypyrrole trilayer actuator displacement," in Electroactive Polymer Actuators and Devices (EAPAD) 2008, March 10, 2008 - March 13, 2008, San Diego, CA, United states, 2008, p. The International Society for Optical Engineering (SPIE); American Society of Mechanical Engineers.

[19] Y. Wu, G. Alici, G. M. Spinks, and G. G. Wallace, "Fast trilayer polypyrrole bending actuators for high speed applications," Synthetic Metals, vol. 156, pp. 1017-1022, 2006.

[20] P. Metz, G. Alici, and G. M. Spinks, "A finite element model for bending behaviour of conducting polymer electromechanical actuators," Sensors and Actuators, A: Physical, vol. 130-131, pp. 1-11, 2006.

[21] S. Yun, J. Kim, and C. Song, "Performance of Electro-active paper actuators with thickness variation," Sensors and Actuators, A: Physical, vol. 133, pp. 225-230, 2007.

[22] R. Minato, "Performance Evaluation of Conducting Polymer Actuators with Different Topologies for Use in Micro/Nano Manipulation Systems," Bachelor of Engineering (Mechatronics), Faculty of Engineering, Wollongong University, Wollongong, 2006.

[23] S. W. John, G. Alici, G. M. Spinks, J. M. D. Madden, and G. G. Wallace, "Towards fully optimised conducting polymer bending sensors: Effect of geometry," Journal of Smart Materials and Structures, 2009.

[24] P. S. Fruehauf, I. L. Chien, and M. D. Lauritsen, "Simplified IMC-PID tuning rules," ISA Transactions, vol. 33, pp. 43-59, 1994.

[25] F. Karray and C. D. Silva, Soft Computing and Tools of Intelligent Systems Design: Theory and Applications: Pearson Wesley, 2004.

[26] T. Takagi and M. Sugeno, "Derivation of Fuzzy Control Rules from Human Operator's Control Actions," Proc. IFAC Symp. Fuzzy Inform., Knowledge Representation and Decision Analysis, pp. 55-60, July 1983.

[27] S. Haykin, Neural Networks: A Comprehensive Foundation: Prentice Hall PTR, 1998.

[28] J. S. R. Jang, "ANFIS - Adaptive-Network-Based Fuzzy Inference System," IEEE Transactions on Systems Man and Cybernetics, vol. 23, pp. 665-685, May-Jun 1993.

[29] G. Alici and N. N. Huynh, "Performance quantification of conducting polymer actuators for real applications: A microgripping system," IEEE/ASME Transactions on Mechatronics, vol. 12, pp. 73-84, 2007. 


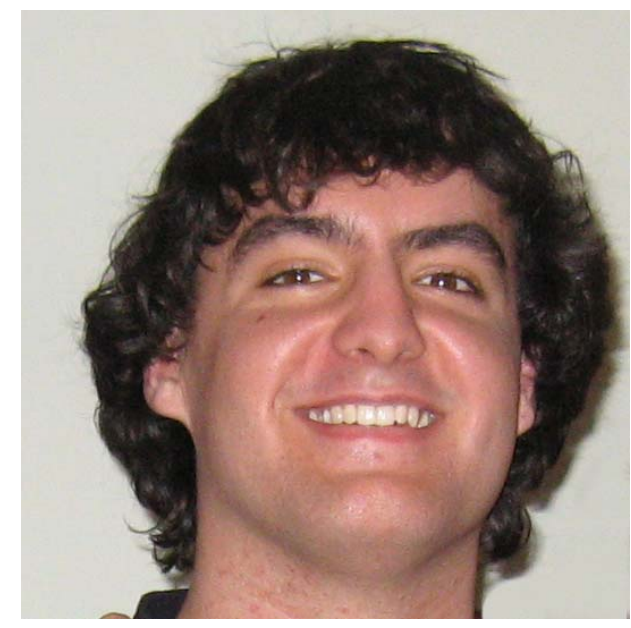

Christopher M. Druitt received a B.E (honors) in mechatronic engineering from the School of Mechanical, Materials and Mechatronic Engineering at the University of Wollongong, Wollongong, Australia, in 2011. He is

now with BAE Systems Australia, North Ryde working as a mechatronic engineer on a variety of naval and combat system projects.

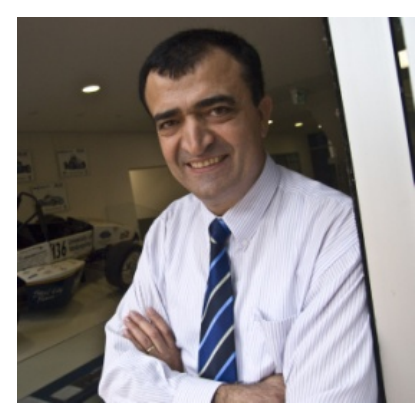

Gursel Alici received a Ph.D. degree in Robotics from the Department of Engineering Science, Oxford University, $\mathrm{UK}$, in 1994. He is currently a Professor at the University of Wollongong, NSW, Australia, where he is the head of School of Mechanical, Materials and Mechatronic Engineering. His current research interests include intelligent mechatronic systems involving mechanisms/serial/parallel robot manipulators, macro/micro/nano robotic systems for medical applications, and conducting polymers as macro/micro/nano sized-actuators and sensors for soft robotic and bio-inspired applications.

He was a technical editor of IEEE/ASME Transactions on Mechatronics during 2008-2012. He is a member of the Mechatronics National Panel formed by the Institution of Engineers, Australia. He has served on the international program committee of numerous IEEE/ASME international conferences on Robotics and Mechatronics. He was the general chair of 2013 IEEE/ASME International Conferences on Advanced Intelligent Mechatronics held in Wollongong, Australia. He is one of the chief investigators of the ARC Center of Excellence for Electromaterials Science (ACES) in the Energy conversion and Bionics programs. He has published more than 220 refereed publications in his areas of research. Gursel Alici is the recipient of the Outstanding Contributions to Teaching and Learning (OCTAL) award in 2010, and 2013 Vice-Chancellor's Interdisciplinary Research Excellence Award from the University of Wollongong. 